\title{
QUANTITATIVE UNIQUE CONTINUATION PRINCIPLE FOR SCHRÖDINGER OPERATORS WITH SINGULAR POTENTIALS
}

\author{
ABEL KLEIN AND C.S. SIDNEY TSANG
}

\begin{abstract}
We prove a quantitative unique continuation principle for Schrödinger operators $H=-\Delta+V$ on $\mathrm{L}^{2}(\Omega)$, where $\Omega$ is an open subset of $\mathbb{R}^{d}$ and $V$ is a singular potential: $V \in \mathrm{L}^{\infty}(\Omega)+\mathrm{L}^{p}(\Omega)$. As an application, we derive a unique continuation principle for spectral projections of Schrödinger operators with singular potentials.
\end{abstract}

\section{IntRoduCtion}

We prove a quantitative unique continuation principle for Schrödinger operators $H=-\Delta+V$ on $\mathrm{L}^{2}(\Omega)$, where $\Omega$ is an open subset of $\mathbb{R}^{d}, \Delta$ is the Laplacian operator, and $V$ is a singular real potential: $V \in \mathrm{L}^{\infty}(\Omega)+\mathrm{L}^{p}(\Omega)$. Our results extend the original result of Bourgain and Kenig [BK] Lemma 3.10], as well as subsequent versions [GK2, Theorem A.1] and [BKl, Theorem 3.4], where $V$ is a bounded potential: $V \in \mathrm{L}^{\infty}(\Omega)$.

As an application, we derive a unique continuation principle for spectral projections of Schrödinger operators with singular potentials, extending the bounded potential results of [Kl, Theorem 1.1] and [KIN, Theorem B.1].

To prove the quantitative unique continuation principle for singular potentials we use Sobolev inequalities (not required for bounded potentials). Since the Sobolev inequality we use in dimension $d=2$ is expressed in terms of Orlicz norms, we review Orlicz spaces, following $\left[\mathbb{R R}\right.$. A function $\varphi: \mathbb{R}^{+} \rightarrow \mathbb{R}^{+} \cup\{+\infty\}$ is called a Young function if it is increasing, convex, $\varphi(0)=0$, and $\lim _{t \rightarrow \infty} \varphi(t)=\infty$. Its complementary function, given by $\varphi^{*}(t)=\sup _{s \in \mathbb{R}^{+}}\{s t-\varphi(s)\}$ for $t \in \mathbb{R}^{+}$, is also a Young function. Given a Young function $\varphi$ and a $\sigma$-finite measure $\mu$ on a measurable space $X$, we define the Orlicz space

$$
\mathrm{L}^{\varphi}(X)=\left\{f: X \rightarrow \mathbb{R} \text { measurable } \mid \int_{X} \varphi(\alpha|f|) d \mu<\infty \text { for some } \alpha>0\right\},
$$

a Banach space when equipped with the Orlicz norm

$$
\|f\|_{\varphi}:=\inf \left\{k>0: \int_{X} \varphi\left(\frac{1}{k}|f|\right) d \mu \leq 1\right\} .
$$

(A standard example is $\varphi(t)=t^{p}$ with $1 \leq p<\infty$; in this case $\mathrm{L}^{\varphi}(X)=\mathrm{L}^{p}(X)$.) There is a Hölder's inequality for Orlicz spaces:

$$
\int_{X}|f g| d \mu \leq 2\|f\|_{\varphi}\|g\|_{\varphi^{*}} \quad \text { for all } \quad f \in \mathrm{L}^{\varphi}(X), g \in \mathrm{L}^{\varphi^{*}}(X) .
$$

2010 Mathematics Subject Classification. 35B99 (Primary), 81Q10 (Secondary).

A.K. and C.S.S.T. were supported by the NSF under grant DMS-1301641. 
We now state our main theorem, a quantitative unique continuation principle for Schrödinger operators with singular potentials. We fix the Young function

$$
\varphi(t)=e^{t}-1, \quad \text { so } \quad \varphi^{*}(t)=\left\{\begin{array}{ll}
0 & \text { if } 0 \leq t \leq 1 \\
t \log t-t+1 & \text { if } t>1
\end{array} .\right.
$$

We use the norm $|x|:=\left(\sum_{j=1}^{d}\left|x_{j}\right|^{2}\right)^{\frac{1}{2}}$ for $x=\left(x_{1}, x_{2}, \ldots, x_{d}\right) \in \mathbb{R}^{d}$; all distances in $\mathbb{R}^{d}$ will be measured with respect to this norm. By $B(x, \delta):=\left\{y \in \mathbb{R}^{d}:|y-x|<\delta\right\}$ we denote the ball centered at $x \in \mathbb{R}^{d}$ with radius $\delta>0$. Given subsets $A$ and $B$ of $\mathbb{R}^{d}$, and a function $\phi$ on set $B$, we set $\phi_{A}:=\phi \chi_{A \cap B}$. We let $\phi_{x, \delta}:=\phi_{B(x, \delta)}$.

Theorem 1.1. Let $\Omega$ be an open subset of $\mathbb{R}^{d}, K=K_{1}+K_{2}$ with $K_{1}, K_{2} \geq 0$, and consider a real measurable function $V=V^{(1)}+V^{(2)}$ on $\Omega$ with $\left\|V^{(1)}\right\|_{\infty} \leq K_{1}$. Let $\psi \in \mathrm{L}^{2}(\Omega)$ be real valued with $\Delta \psi \in \mathrm{L}_{\text {loc }}^{2}(\Omega)$, and suppose

$$
\zeta=-\Delta \psi+V \psi \in \mathrm{L}^{2}(\Omega) \text {. }
$$

Fix a bounded measurable set $\Theta \subset \Omega$ where $\left\|\psi_{\Theta}\right\|_{2}>0$, and set

$$
Q(x, \Theta):=\sup _{y \in \Theta}|y-x| \quad \text { for } \quad x \in \Omega .
$$

Consider $x_{0} \in \Omega \backslash \bar{\Theta}$ such that

$$
Q=Q\left(x_{0}, \Theta\right) \geq 1 \quad \text { and } B\left(x_{0}, 6 Q+2\right) \subset \Omega,
$$

and take

$$
0<\delta \leq \min \left\{\operatorname{dist}\left(x_{0}, \Theta\right), \frac{1}{2}\right\} .
$$

There is a constant $m_{d}>0$, depending only on $d$, such that:

(i) If either $d \geq 3$ and $\left\|V^{(2)}\right\|_{p} \leq K_{2}$ with $p \geq d$, or $d=2$ and $\left(\left\|\left|V^{(2)}\right|^{p}\right\|_{\varphi^{*}}\right)^{\frac{1}{p}} \leq$ $K_{2}$ with $p \geq 2$, we have

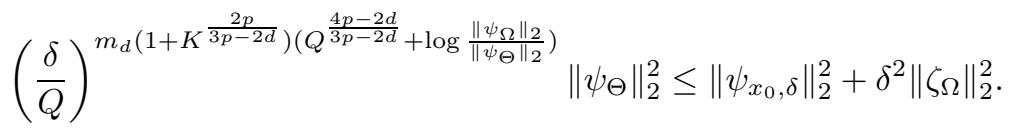

In particular, if $d=2$ it suffices to require $\left\|V^{(2)}\right\|_{p} \leq K_{2}$ with $p>2$ to obtain (1.9).

(ii) If $d=1$ and $\left\|V^{(2)}\right\|_{p} \leq K_{2}$ with $p \geq 2$, we have

$$
\left(\frac{\delta}{Q}\right)^{m_{1}\left(1+K^{\frac{2 p}{3 p-4}}\right)\left(Q^{\frac{4 p-4}{3 p-4}}+\log \frac{\left\|\psi_{\Omega}\right\|_{2}}{\left\|\psi_{\Theta}\right\|_{2}}\right)}\left\|\psi_{\Theta}\right\|_{2}^{2} \leq\left\|\psi_{x_{0}, \delta}\right\|_{2}^{2}+\delta^{2}\left\|\zeta_{\Omega}\right\|_{2}^{2}
$$

Letting $p \rightarrow \infty$ in Theorem 1.1 we recover [BKl, Theorem 3.4]. The proof of Theorem 1.1, given in Section 2] relies on a Carleman estimate of Escauriaza and Vesella EV, Theorem 2], stated in Lemma 2.1. To control singular potentials we use all the terms in this estimate, including the the gradient term, and Sobolev's inequalities. In the proofs for bounded potentials BK, GK2, BKl] it suffices to use a simpler version of this Carleman estimate without the the gradient term (see BK, Lemma 3.15]).

As an application of Theorem 1.1, we prove a unique continuation principle for spectral projections of Schrödinger operators with singular potentials, extending [Kl, Theorem 1.1] (in the form given in [KIN, Theorem B.1]) to Schrödinger operators with singular potentials. (See also [CHK1, Section 4], CHK2, Theorem 2.1], 
GK2, Theorem A.6], and [RoV, Theorem 2.1] for unique continuation principles for spectral projections of Schrödinger operators with bounded potentials.)

We consider rectangles in $\mathbb{R}^{d}$ of the form

$$
\Lambda=\Lambda_{\mathbf{L}}(a)=a+\prod_{j=1}^{d}\left(-\frac{L_{j}}{2}, \frac{L_{j}}{2}\right)=\prod_{j=1}^{d}\left(a_{j}-\frac{L_{j}}{2}, a_{j}+\frac{L_{j}}{2}\right),
$$

where $a=\left(a_{1}, \ldots, a_{d}\right) \in \mathbb{R}^{d}$ and $\mathbf{L}=\left(L_{1}, \ldots, L_{d}\right) \in(0, \infty)^{d}$. (We write $\Lambda_{L}(a)=$ $\Lambda_{\mathbf{L}}(a)$ in the special case $L_{j}=L$ for $j=1, \ldots, d$.) Given a Schrödinger operator $H=-\Delta+V$ on $\mathrm{L}^{2}\left(\mathbb{R}^{d}\right)$, by $H_{\Lambda}=-\Delta_{\Lambda}+V_{\Lambda}$ we denote the restriction of $H$ to the rectangle $\Lambda$ with either Dirichlet or periodic boundary condition: $\Delta_{\Lambda}$ is the Laplacian on $\Lambda$ with either Dirichlet or periodic boundary condition, and $V_{\Lambda}$ is the restriction of $V$ to $\Lambda$.

Theorem 1.2. Let $H=-\Delta+V$ be a Schrödinger operator on $\mathrm{L}^{2}\left(\mathbb{R}^{d}\right)$, where $V=V^{(1)}+V^{(2)}$ with $\left\|V^{(1)}\right\|_{\infty} \leq K_{1}<\infty$ and $\left\|V^{(2)}\right\|_{p} \leq K_{2}<\infty$ with $p \geq d$ for $d \geq 3, p>2$ for $d=2$, and $p \geq 2$ for $d=1$. Set $K=K_{1}+K_{2}$. Fix $\delta \in\left(0, \frac{1}{2}\right]$, and let $\left\{y_{k}\right\}_{k \in \mathbb{Z}^{d}}$ be sites in $\mathbb{R}^{d}$ with $B\left(y_{k}, \delta\right) \subset \Lambda_{1}(k)$ for all $k \in \mathbb{Z}^{d}$. There exists a constant $M_{d}>0$, depending only on $d$, such that, defining $\gamma=\gamma\left(d, p, K, \delta, E_{0}\right)>0$ for $E_{0}>0$ by

$$
\gamma^{2}=\left\{\begin{array}{ll}
\frac{1}{2} \delta^{M_{d}\left(1+\left(K+E_{0}\right)^{\frac{4 p^{2}}{(3 p-2 d)(2 p-d)}}\right)} & \text { for } \quad d \geq 2 \\
\frac{1}{2} \delta^{M_{d}\left(1+\left(K+E_{0}\right)^{\frac{2 p^{2}}{(3 p-4)(p-1)}}\right)} & \text { for } \quad d=1
\end{array},\right.
$$

then, given a rectangle $\Lambda$ as in (1.11), where $a \in \mathbb{R}^{d}$ and $L_{j} \geq 114 \sqrt{d}$ for $j=$ $1, \ldots, d$, and a closed interval $I \subset\left(-\infty, E_{0}\right]$ with $|I| \leq 2 \gamma$, we have

$$
\chi_{I}\left(H_{\Lambda}\right) W^{(\Lambda)} \chi_{I}\left(H_{\Lambda}\right) \geq \gamma^{2} \chi_{I}\left(H_{\Lambda}\right),
$$

where

$$
W^{(\Lambda)}=\sum_{k \in \mathbb{Z}^{d}, \Lambda_{1}(k) \subset \Lambda} \chi_{B\left(y_{k}, \delta\right)} .
$$

The proof of Theorem 1.2 is discussed in Section 3

Remark 1.3. Using Theorem 1.2 we can prove optimal Wegner estimates for Anderson Hamiltonians with singular background potentials, extending the results of Kl].

\section{Quantitative Unique CONTINUation PRINCIPLE FOR Schrödinger OPERATORS WITH SINGULAR POTENTIALS}

The proof of Theorem 1.1 is based on a Carleman estimate of Escauriaza and Vesella [EV, Theorem 2], which we state in a ball of radius $\varrho>0$.

Lemma 2.1. Given $\varrho>0$, the function $\omega_{\varrho}(x)=\phi\left(\frac{1}{\varrho}|x|\right)$ on $\mathbb{R}^{d}$, where $\phi(s):=$ $s e^{-\int_{0}^{s} \frac{1-e^{-t}}{t} d t}$, is a strictly increasing continuous function on $[0, \infty), \mathrm{C}^{\infty}$ on $(0, \infty)$, satisfying

$$
\frac{1}{C_{1} \varrho}|x| \leq \omega_{\varrho}(x) \leq \frac{1}{\varrho}|x| \quad \text { for } \quad x \in B(0, \varrho),
$$


where $C_{1}=\phi(1)^{-1} \in(2,3)$. Moreover, there exist positive contants $C_{2}$ and $C_{3}$, depending only on $d$, such that for all $\alpha \geq C_{2}$ and all real valued functions $f \in$ $H^{2}(B(0, \varrho))$ with $\operatorname{supp} f \subset B(0, \varrho) \backslash\{0\}$ we have

$$
\alpha^{3} \int_{\mathbb{R}^{d}} \omega_{\varrho}^{-1-2 \alpha} f^{2} d x+\alpha \varrho^{2} \int_{\mathbb{R}^{d}} \omega_{\varrho}^{1-2 \alpha}|\nabla f|^{2} d x \leq C_{3} \varrho^{4} \int_{\mathbb{R}^{d}} \omega_{\varrho}^{2-2 \alpha}(\Delta f)^{2} d x .
$$

This estimate is given in the parabolic setting in EV], but the estimate in the elliptic setting as in the lemma follows immediately by the argument in KSU, Proposition B.3]. In the proofs of the quantitative unique continuation principle for bounded potentials BK, GK2, BKl only the first term in the left hand side of (2.2) is used (see BK, Lemma 3.15]), but for singular potentials we also need to use the gradient term in the left hand side of (2.2) and Sobolev's inequalities.

Proof of Theorem 1.1. Let $C_{1}, C_{2}, C_{3}$ be the constants of Lemma 2.1, which depend only on $d$. Without loss of generality $C_{2}>1$. By $C_{j}, j=4,5, \ldots$, we will always denote an appropriate nonzero constant depending only on $d$.

We follow Bourgain and Klein's proof for bounded potentials [BKl, Theorem 3.4]. Let $x_{0} \in \Omega \backslash \bar{\Theta}$ be as in (1.7). Without loss of generality we take $x_{0}=0, \Theta \subset$ $B\left(0,2 C_{1} Q\right)$, and $\Omega=B(0, \varrho)$, where $\varrho=2 C_{1} Q+2$, and let $\delta$ be as in (1.8). Proceeding as in BKl, Theorem 3.4], we fix a function $\eta \in \mathrm{C}_{c}^{\infty}\left(\mathbb{R}^{d}\right)$ given by $\eta(x)=\xi(|x|)$, where $\xi$ is an even $\mathrm{C}^{\infty}$ function on $\mathbb{R}, 0 \leq \xi \leq 1$, such that

$$
\begin{aligned}
& \xi(s)=1 \quad \text { if } \quad \frac{3}{4} \delta \leq|s| \leq 2 C_{1} Q, \quad \xi(s)=0 \quad \text { if } \quad|s| \leq \frac{1}{4} \delta \text { or }|s| \geq 2 C_{1} Q+1, \\
& \left|\xi^{j}(s)\right| \leq\left(\frac{4}{\delta}\right)^{j} \quad \text { if } \quad|s| \leq \frac{3}{4} \delta, \quad\left|\xi^{j}(s)\right| \leq 2^{j} \quad \text { if } \quad|s| \geq 2 C_{1} Q, j=1,2, \\
& |\nabla \eta(x)| \leq \sqrt{d}\left|\xi^{\prime}(|x|)\right| \quad \text { and } \quad|\Delta \eta(x)| \leq d\left|\xi^{\prime \prime}(|x|)\right|, \\
& \operatorname{supp} \nabla \eta \subset\left\{\frac{\delta}{4} \leq|x| \leq \frac{3 \delta}{4}\right\} \cup\left\{2 C_{1} Q \leq|x| \leq 2 C_{1} Q+1\right\}
\end{aligned}
$$

Let $\alpha \geq C_{2}$. Applying Lemma 2.1 to the function $\eta \psi$ gives

$$
\begin{aligned}
\frac{\alpha^{3}}{3 C_{3} \varrho^{4}} & \int_{\mathbb{R}^{d}} \omega_{\varrho}^{-1-2 \alpha} \eta^{2} \psi^{2} d x+\frac{\alpha}{3 C_{3} \varrho^{2}} \int_{\mathbb{R}^{d}} \omega_{\varrho}^{1-2 \alpha}|\nabla(\eta \psi)|^{2} d x \\
\leq & \frac{1}{3} \int_{\mathbb{R}^{d}} \omega_{\varrho}^{2-2 \alpha}(\Delta(\eta \psi))^{2} d x \leq \int_{\mathbb{R}^{d}} \omega_{\varrho}^{2-2 \alpha} \eta^{2}(\Delta \psi)^{2} d x \\
& +4 \int_{\operatorname{supp} \nabla \eta} \omega_{\varrho}^{2-2 \alpha}|\nabla \eta|^{2}|\nabla \psi|^{2} d x+\int_{\operatorname{supp} \nabla \eta} \omega_{\varrho}^{2-2 \alpha}(\Delta \eta)^{2} \psi^{2} d x
\end{aligned}
$$

Using (1.5), $\left\|V^{(1)}\right\|_{\infty} \leq K_{1}$, and $\omega_{\varrho} \leq 1$ on supp $\eta$, we have

$$
\begin{aligned}
& \int_{\mathbb{R}^{d}} \omega_{\varrho}^{2-2 \alpha} \eta^{2}(\Delta \psi)^{2} d x \leq 2 \int_{\mathbb{R}^{d}} V^{2} \omega_{\varrho}^{2-2 \alpha} \eta^{2} \psi^{2} d x+2 \int_{\mathbb{R}^{d}} \omega_{\varrho}^{2-2 \alpha} \eta^{2} \zeta^{2} d x \\
& \quad \leq 4 K_{1}^{2} \int_{\mathbb{R}^{d}} \omega_{\varrho}^{-1-2 \alpha} \eta^{2} \psi^{2} d x+4 \int_{\mathbb{R}^{d}}\left(V^{(2)}\right)^{2} \omega_{\varrho}^{2-2 \alpha} \eta^{2} \psi^{2} d x+2 \int_{\mathbb{R}^{d}} \omega_{\varrho}^{2-2 \alpha} \eta^{2} \zeta^{2} d x .
\end{aligned}
$$

Given $M>0$, we write $V^{(2)}=U_{M}+V_{M}$, where $U_{M}=V^{(2)} \chi_{\left\{\left|V^{(2)}\right| \leq \sqrt{M}\right\}}$ and $W_{M}=V^{(2)} \chi_{\left\{\left|V^{(2)}\right|>\sqrt{M}\right\}}$. We have

$$
\int_{\mathbb{R}^{d}}\left(V^{(2)}\right)^{2} \omega_{\varrho}^{2-2 \alpha} \eta^{2} \psi^{2} d x \leq M \int_{\mathbb{R}^{d}} \omega_{\varrho}^{-1-2 \alpha} \eta^{2} \psi^{2} d x+\int_{\mathbb{R}^{d}} W_{M}^{2} \omega_{\varrho}^{2-2 \alpha} \eta^{2} \psi^{2} d x .
$$


Combining (2.4), (2.5) and (2.6), we have

$$
\begin{aligned}
& \left(\frac{\alpha^{3}}{3 C_{3} \varrho^{4}}-4 K_{1}^{2}-4 M\right) \int_{\mathbb{R}^{d}} \omega_{\varrho}^{-1-2 \alpha} \eta^{2} \psi^{2} d x+\frac{\alpha}{3 C_{3} \varrho^{2}} \int_{\mathbb{R}^{d}} \omega_{\varrho}^{1-2 \alpha}|\nabla(\eta \psi)|^{2} d x \\
& \quad \leq 4 \int_{\mathbb{R}^{d}} W_{M}^{2} \omega_{\varrho}^{2-2 \alpha} \eta^{2} \psi^{2} d x+2 \int_{\mathbb{R}^{d}} \omega_{\varrho}^{2-2 \alpha} \eta^{2} \zeta^{2} d x \\
& \quad+4 \int_{\operatorname{supp} \nabla \eta} \omega_{\varrho}^{2-2 \alpha}|\nabla \eta|^{2}|\nabla \psi|^{2} d x+\int_{\operatorname{supp} \nabla \eta} \omega_{\varrho}^{2-2 \alpha}(\Delta \eta)^{2} \psi^{2} d x .
\end{aligned}
$$

Note that for $1 \leq q \leq p$ we have

$$
\left\|W_{M}\right\|_{q} \leq M^{-\frac{p-q}{2 q}}\left\|W_{M}\right\|_{p}^{\frac{p}{q}} \leq M^{-\frac{p-q}{2 q}}\left\|V^{(2)}\right\|_{p}^{\frac{p}{q}} \leq M^{-\frac{p-q}{2 q}} K_{2}^{\frac{p}{q}} .
$$

We set $K=K_{1}+K_{2}$ with $K_{1}, K_{2} \geq 0$.

We consider three cases:

(a) $d \geq 3$ : Let $\left\|V^{(2)}\right\|_{p} \leq K_{2}$ with $p \geq d$. Using Hölder's inequality and (2.8) with $q=d$, we get

$$
\begin{aligned}
& \int_{\mathbb{R}^{d}} W_{M}^{2} \omega_{\varrho}^{2-2 \alpha} \eta^{2} \psi^{2} d x \leq\left\|W_{M}^{2}\right\|_{\frac{d}{2}}\left\|\omega_{\varrho}^{2-2 \alpha} \eta^{2} \psi^{2}\right\|_{\frac{d}{d-2}} \\
& =\left\|W_{M}\right\|_{d}^{2}\left\|\omega_{\varrho}^{1-\alpha} \eta \psi\right\|_{\frac{2 d}{d-2}}^{2} \leq M^{-\frac{p-d}{d}} K_{2}^{\frac{2 p}{d}}\left\|\omega_{\varrho}^{1-\alpha} \eta \psi\right\|_{\frac{2 d}{d-2}}^{2} .
\end{aligned}
$$

Using Sobolev's inequality (e.g., [GiT, Theorem 7.10]), we get

$$
\begin{aligned}
\left\|\omega_{\varrho}^{1-\alpha} \eta \psi\right\|_{\frac{2 d}{d-2}}^{2} & \leq C_{4}\left(\int_{\mathbb{R}^{d}}\left|\nabla\left(\omega_{\varrho}^{1-\alpha} \eta \psi\right)\right|^{2}\right) \\
& \leq 2 C_{4} \int_{\mathbb{R}^{d}}\left|\nabla \omega_{\varrho}^{1-\alpha}\right|^{2} \eta^{2} \psi^{2} d x+2 C_{4} \int_{\mathbb{R}^{d}} \omega_{\varrho}^{1-2 \alpha}|\nabla(\eta \psi)|^{2} d x .
\end{aligned}
$$

Since

$$
\left|\nabla \omega_{\varrho}^{1-\alpha}\right|^{2}=(1-\alpha)^{2} \frac{\omega_{\varrho}^{2-2 \alpha}}{|x|^{2} \exp \left(\frac{2}{\varrho}|x|\right)} \leq \frac{\alpha^{2}}{\varrho^{2}} \omega_{\varrho}^{-2 \alpha},
$$

we have (recall $\omega_{\varrho} \leq 1$ on $\left.\operatorname{supp} \eta\right)$

$$
\int_{\mathbb{R}^{d}}\left|\nabla \omega_{\varrho}^{1-\alpha}\right|^{2} \eta^{2} \psi^{2} d x \leq \frac{\alpha^{2}}{\varrho^{2}} \int_{\mathbb{R}^{d}} \omega_{\varrho}^{-1-2 \alpha} \eta^{2} \psi^{2} d x .
$$

Combining (2.7), (2.9), (2.10) and (2.12), we conclude that

$$
\begin{gathered}
\left(\frac{\alpha^{3}}{3 C_{3} \varrho^{4}}-4 K_{1}^{2}-4 M-8 C_{4} M^{-\frac{p-d}{d}} K_{2}^{\frac{2 p}{d}} \frac{\alpha^{2}}{\varrho^{2}}\right) \int_{\mathbb{R}^{d}} \omega_{\varrho}^{-1-2 \alpha} \eta^{2} \psi^{2} d x \\
+\left(\frac{\alpha}{3 C_{3} \varrho^{2}}-8 C_{4} M^{-\frac{p-d}{d}} K_{2}^{\frac{2 p}{d}}\right) \int_{\mathbb{R}^{d}} \omega_{\varrho}^{1-2 \alpha}|\nabla(\eta \psi)|^{2} d x \\
\leq 4 \int_{\operatorname{supp} \nabla \eta} \omega_{\varrho}^{2-2 \alpha}|\nabla \eta|^{2}|\nabla \psi|^{2} d x+\int_{\operatorname{supp} \nabla \eta} \omega_{\varrho}^{2-2 \alpha}(\Delta \eta)^{2} \psi^{2} d x \\
+2 \int_{\operatorname{supp} \eta} \omega_{\varrho}^{2-2 \alpha} \eta^{2} \zeta^{2} d x .
\end{gathered}
$$


Assuming $\alpha \geq \varrho$ and setting $M=K_{2}^{2} \alpha^{\frac{2 d}{p}} \varrho^{\frac{-2 d}{p}}$, we have

$$
\begin{aligned}
4 K_{1}^{2}+4 M+8 C_{4} M^{-\frac{p-d}{d}} K_{2}^{\frac{2 p}{d}} \alpha^{2} \varrho^{-2} & =4 K_{1}^{2}+4 K_{2}^{2}\left(1+2 C_{4}\right) \alpha^{\frac{2 d}{p}} \varrho^{\frac{-2 d}{p}} \\
& \leq\left(4 K^{2}\left(1+2 C_{4}\right)\right) \alpha^{\frac{2 d}{p}} \varrho^{\frac{-2 d}{p}} .
\end{aligned}
$$

Taking

$$
\alpha \geq C_{5}\left(1+K^{\frac{2 p}{3 p-2 d}}\right) \varrho^{\frac{4 p-2 d}{3 p-2 d}} \geq C_{5}\left(1+K^{\frac{2 p}{3 p-2 d}}\right) \varrho^{\frac{4}{3}},
$$

we can guarantee that $\alpha>C_{2}$,

$$
\frac{\alpha^{3}}{3 C_{3} \varrho^{4}} \geq 3\left(4 K^{2}\left(1+2 C_{4}\right) \alpha^{\frac{2 d}{p}} \varrho^{\frac{-2 d}{p}}\right),
$$

and

$$
\frac{\alpha}{3 C_{3} \varrho^{2}}-8 C_{4} M^{-\frac{p-d}{d}} K_{2}^{\frac{2 p}{d}} \geq 0 .
$$

Using (2.1) and recalling (1.6), we obtain

$$
\int_{\mathbb{R}^{d}} \omega_{\varrho}^{-1-2 \alpha} \eta^{2} \psi^{2} d x \geq\left(\frac{\varrho}{Q}\right)^{1+2 \alpha}\left\|\psi_{\Theta}\right\|_{2}^{2} \geq\left(2 C_{1}\right)^{1+2 \alpha}\left\|\psi_{\Theta}\right\|_{2}^{2} .
$$

Combining (2.13), (2.16), (2.17) and (2.18), we conclude that

$$
\begin{aligned}
\frac{2 \alpha^{3}}{9 C_{3} \varrho^{4}}\left(2 C_{1}\right)^{1+2 \alpha}\left\|\psi_{\Theta}\right\|_{2}^{2} \leq 4 \int_{\operatorname{supp} \nabla \eta} \omega_{\varrho}^{2-2 \alpha}|\nabla \eta|^{2}|\nabla \psi|^{2} d x \\
+\int_{\operatorname{supp} \nabla \eta} \omega_{\varrho}^{2-2 \alpha}(\Delta \eta)^{2} \psi^{2} d x+2 \int_{\operatorname{supp} \eta} \omega_{\varrho}^{2-2 \alpha} \eta^{2} \zeta^{2} d x .
\end{aligned}
$$

Let $f \in \mathcal{D}(\nabla)$. For arbitrary $M>0$ we have

$$
\left|\int_{\mathbb{R}^{d}} V f^{2} d x\right| \leq\left(K_{1}+M^{\frac{1}{2}}\right)\|f\|_{2}^{2}+\int_{\mathbb{R}^{d}}\left|W_{M}\right| f^{2} d x .
$$

Using Hölder's inequality, (2.8) with $q=\frac{d}{2}$, and Sobolev's inequality, we get

$$
\left|\int_{\mathbb{R}^{d}} V f^{2} d x\right| \leq\left(K_{1}+M^{\frac{1}{2}}\right)\|f\|_{2}^{2}+C_{4} M^{-\frac{2 p-d}{2 d}} K_{2}^{\frac{2 p}{d}}\|\nabla f\|_{2}^{2} .
$$

Taking $M=\left(2 C_{4} K_{2}^{\frac{2 p}{d}}\right)^{\frac{2 d}{2 p-d}}$ (we can require $C_{4} \geq 1$ ), we get

$$
\left|\int_{\mathbb{R}^{d}} V f^{2} d x\right| \leq 2 C_{4}\left(1+K^{\frac{2 p}{2 p-d}}\right)\|f\|_{2}^{2}+\frac{1}{2}\|\nabla f\|_{2}^{2} .
$$

We have

$$
\begin{aligned}
& \int_{\left\{2 C_{1} Q \leq|x| \leq 2 C_{1} Q+1\right\}} \omega_{\varrho}^{2-2 \alpha}\left(4|\nabla \eta|^{2}|\nabla \psi|^{2}+(\Delta \eta)^{2} \psi^{2}\right) d x \\
& \quad \leq 16 d^{2}\left(\frac{C_{1} \varrho}{2 C_{1} Q}\right)^{2 \alpha-2} \int_{\left\{2 C_{1} Q \leq|x| \leq 2 C_{1} Q+1\right\}}\left(4|\nabla \psi|^{2}+\psi^{2}\right) d x \\
& \quad \leq C_{6}\left(\frac{5}{4} C_{1}\right)^{2 \alpha-2} \int_{\left\{2 C_{1} Q-1 \leq|x| \leq 2 C_{1} Q+2\right\}}\left(\zeta^{2}+\left(1+K^{\frac{2 p}{2 p-d}}\right) \psi^{2}\right) d x \\
& \quad \leq C_{6}\left(\frac{5}{4} C_{1}\right)^{2 \alpha-2}\left(\left\|\zeta_{\Omega}\right\|_{2}^{2}+\left(1+K^{\frac{2 p}{2 p-d}}\right)\left\|\psi_{\Omega}\right\|_{2}^{2}\right),
\end{aligned}
$$


where we used (2.22) and an interior estimate (e.g., GK1, Lemma A.2]). Similarly,

$$
\begin{aligned}
& \int_{\left\{\frac{\delta}{4} \leq|x| \leq \frac{3 \delta}{4}\right\}} \omega_{\varrho}^{2-2 \alpha}\left(4|\nabla \eta|^{2}|\nabla \psi|^{2}+(\Delta \eta)^{2} \psi^{2}\right) d x \\
& \quad \leq 256 d^{2} \delta^{-4}\left(4 \delta^{-1} C_{1} \varrho\right)^{2 \alpha-2} \int_{\left\{\frac{\delta}{4} \leq|x| \leq \frac{3 \delta}{4}\right\}}\left(4|\nabla \psi|^{2}+\psi^{2}\right) d x \\
& \quad \leq C_{7} \delta^{-4}\left(4 \delta^{-1} C_{1} \varrho\right)^{2 \alpha-2} \int_{\{|x| \leq \delta\}}\left(\zeta^{2}+\left(K^{\frac{2 p}{2 p-d}}+\delta^{-2}\right) \psi^{2}\right) d x \\
& \quad \leq C_{7} \delta^{-4}\left(16 \delta^{-1} C_{1}^{2} Q\right)^{2 \alpha-2}\left(\left\|\zeta_{\Omega}\right\|_{2}^{2}+\left(K^{\frac{2 p}{2 p-d}}+\delta^{-2}\right)\left\|\psi_{0, \delta}\right\|_{2}^{2}\right) .
\end{aligned}
$$

In addition,

$$
\int_{\operatorname{supp} \eta} \omega_{\varrho}^{2-2 \alpha} \eta^{2} \zeta^{2} d x \leq\left(4 \delta^{-1} C_{1} \varrho\right)^{2 \alpha-2}\left\|\zeta_{\Omega}\right\|_{2}^{2} \leq\left(16 \delta^{-1} C_{1}^{2} Q\right)^{2 \alpha-2}\left\|\zeta_{\Omega}\right\|_{2}^{2} .
$$

If we have

$$
\frac{\alpha^{3}}{\varrho^{4}}\left(\frac{8}{5}\right)^{2 \alpha}\left\|\psi_{\Theta}\right\|_{2}^{2} \geq C_{8}\left(1+K^{\frac{2 p}{2 p-d}}\right)\left\|\psi_{\Omega}\right\|_{2}^{2}
$$

we obtain

$$
C_{6}\left(\frac{5}{4} C_{1}\right)^{2 \alpha-2}\left(1+K^{\frac{2 p}{2 p-d}}\right)\left\|\psi_{\Omega}\right\|_{2}^{2} \leq \frac{1}{2} \frac{2 \alpha^{3}}{9 C_{3} \varrho^{4}}\left(2 C_{1}\right)^{1+2 \alpha}\left\|\psi_{\Theta}\right\|_{2}^{2},
$$

so we conclude that

$$
\begin{aligned}
& \frac{\alpha^{3}}{9 C_{3} \varrho^{4}}\left(2 C_{1}\right)^{1+2 \alpha}\left\|\psi_{\Theta}\right\|_{2}^{2} \\
& \quad \leq C_{9} \delta^{-4}\left(16 \delta^{-1} C_{1}^{2} Q\right)^{2 \alpha-2}\left(\left(K^{\frac{2 p}{2 p-d}}+\delta^{-2}\right)\left\|\psi_{0, \delta}\right\|_{2}^{2}+\left\|\zeta_{\Omega}\right\|_{2}^{2}\right) .
\end{aligned}
$$

Thus,

$$
\frac{\alpha^{3}}{\varrho^{4}} Q^{4}\left(\left(8 C_{1} Q\right)^{-1} \delta\right)^{2 \alpha+2}\left\|\psi_{\Theta}\right\|_{2}^{2} \leq C_{10}\left(\left(K^{\frac{2 p}{2 p-d}}+\delta^{-2}\right)\left\|\psi_{0, \delta}\right\|_{2}^{2}+\left\|\zeta_{\Omega}\right\|_{2}^{2}\right) .
$$

Since $\left(\frac{\delta}{Q}\right)^{5} \leq\left(\frac{1}{2}\right)^{5} \leq \frac{1}{8 C_{1}}$ by (1.8), we have

$$
\frac{\alpha^{3}}{\varrho^{4}} Q^{6}\left(\frac{\delta}{Q}\right)^{12 \alpha+14}\left\|\psi_{\Theta}\right\|_{2}^{2} \leq C_{11}\left(\left(1+K^{\frac{2 p}{2 p-d}}\right)\left\|\psi_{0, \delta}\right\|_{2}^{2}+\delta^{2}\left\|\zeta_{\Omega}\right\|_{2}^{2}\right) .
$$

To satisfy (2.15) and (2.26), we choose

$$
\alpha=C_{12}\left(1+K^{\frac{2 p}{3 p-2 d}}\right)\left(Q^{\frac{4 p-2 d}{3 p-2 d}}+\log \frac{\left\|\psi_{\Omega}\right\|_{2}}{\left\|\psi_{\Theta}\right\|_{2}}\right),
$$

Combining with (2.30), and recalling $Q \geq 1$, we get

$$
\begin{gathered}
\left(1+K^{\frac{2 p}{3 p-2 d}}\right)^{3}\left(\frac{\delta}{Q}\right)^{C_{13}\left(1+K^{\frac{2 p}{3 p-2 d}}\right)\left(Q^{\frac{4 p-2 d}{3 p-2 d}}+\log \frac{\left\|\psi_{\Omega}\right\|_{2}}{\left\|\psi_{\Theta}\right\|_{2}}\right)}\left\|\psi_{\Theta}\right\|_{2}^{2} \\
\leq C_{14}\left(\left(1+K^{\frac{2 p}{2 p-d}}\right)\left\|\psi_{0, \delta}\right\|_{2}^{2}+\delta^{2}\left\|\zeta_{\Omega}\right\|_{2}^{2}\right),
\end{gathered}
$$

and hence

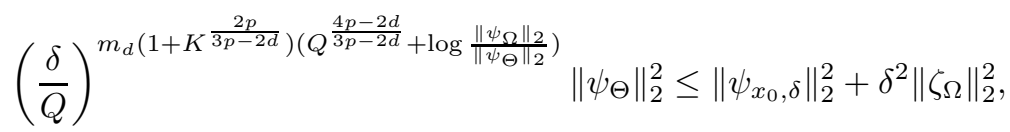

where $m_{d}>0$ is a constant depending only on $d$. 
(b) $d=2$ : Let $\left(\left\|\left|V^{(2)}\right|^{p}\right\|_{\varphi^{*}}\right)^{\frac{1}{p}} \leq K_{2}$ with $p \geq 2$. Given $K_{2}>0$ and $M>0$, we have

$$
\int_{\mathbb{R}^{2}} \varphi^{*}\left(\frac{\left|W_{M}^{2}\right|}{M^{-\frac{p-2}{2}} K_{2}^{p}}\right) d x \leq \int_{\mathbb{R}^{2}} \varphi^{*}\left(\frac{\left|V^{(2)}\right|^{p}}{K_{2}^{p}}\right) d x,
$$

and hence, using $\left\|\left|V^{(2)}\right|^{p}\right\|_{\varphi^{*}} \leq K_{2}^{p}$, we get

$$
\left\|W_{M}^{2}\right\|_{\varphi^{*}} \leq M^{-\frac{p-2}{2}} K_{2}^{p} .
$$

Using Hölder's inequality for Orlicz spaces (1.3), and (2.35), we get

$$
\begin{aligned}
\int_{\mathbb{R}^{2}} W_{M}^{2} \omega_{\varrho}^{2-2 \alpha} \eta^{2} \psi^{2} d x & \leq 2\left\|W_{M}^{2}\right\|_{\varphi^{*}}\left\|\omega_{\varrho}^{2-2 \alpha} \eta^{2} \psi^{2}\right\|_{\varphi} \\
& \leq 2 M^{-\frac{p-2}{2}} K_{2}^{p}\left\|\omega_{\varrho}^{2-2 \alpha} \eta^{2} \psi^{2}\right\|_{\varphi} .
\end{aligned}
$$

Using the Sobolev inequality given in [AT, Theorem 0.1], we obtain

$$
\begin{aligned}
\left\|\omega_{\varrho}^{2-2 \alpha} \eta^{2} \psi^{2}\right\|_{\varphi} \leq & C_{4}\left(\int_{\mathbb{R}^{2}}\left|\omega_{\varrho}^{1-\alpha} \eta \psi\right|^{2} d x+\int_{\mathbb{R}^{2}}\left|\nabla\left(\omega_{\varrho}^{1-\alpha} \eta \psi\right)\right|^{2} d x\right) \\
\leq & C_{4} \int_{\mathbb{R}^{2}}\left|\omega_{\varrho}^{1-\alpha} \eta \psi\right|^{2} d x+2 C_{4} \int_{\mathbb{R}^{2}}\left|\nabla \omega_{\varrho}^{1-\alpha}\right|^{2} \eta^{2} \psi^{2} d x \\
& +2 C_{4} \int_{\mathbb{R}^{2}} \omega_{\varrho}^{1-2 \alpha}|\nabla(\eta \psi)|^{2} d x .
\end{aligned}
$$

Combining (2.7), (2.36), (2.37), and (2.12) with $d=2$, we conclude that

$$
\begin{gathered}
\left(\frac{\alpha^{3}}{3 C_{3} \varrho^{4}}-4 K_{1}^{2}-4 M-8 C_{4} M^{-\frac{p-2}{2}} K_{2}^{p}-16 C_{4} M^{-\frac{p-2}{2}} K_{2}^{p} \frac{\alpha^{2}}{\varrho^{2}}\right) \int_{\mathbb{R}^{2}} \omega_{\varrho}^{-1-2 \alpha} \eta^{2} \psi^{2} d x \\
+\left(\frac{\alpha}{3 C_{3} \varrho^{2}}-16 C_{4} M^{-\frac{p-2}{2}} K_{2}^{p}\right) \int_{\mathbb{R}^{2}} \omega_{\varrho}^{1-2 \alpha}|\nabla(\eta \psi)|^{2} d x
\end{gathered}
$$

$$
\begin{aligned}
\leq 4 \int_{\operatorname{supp} \nabla \eta} & \omega_{\varrho}^{2-2 \alpha}|\nabla \eta|^{2}|\nabla \psi|^{2} d x+\int_{\operatorname{supp} \nabla \eta} \omega_{\varrho}^{2-2 \alpha}(\Delta \eta)^{2} \psi^{2} d x \\
& +2 \int_{\operatorname{supp} \eta} \omega_{\varrho}^{2-2 \alpha} \eta^{2} \zeta^{2} d x .
\end{aligned}
$$

Assuming $\alpha \geq \varrho$ and setting $M=K_{2}^{2} \alpha^{\frac{4}{p}} \varrho^{-\frac{4}{p}}$, we have

$$
\begin{aligned}
& 4 K_{1}^{2}+4 M+8 C_{4} M^{-\frac{p-2}{2}} K_{2}^{p}+16 C_{4} M^{-\frac{p-2}{2}} K_{2}^{p} \frac{\alpha^{2}}{\varrho^{2}} \\
& \quad \leq 4 K_{1}^{2}+4 M+24 C_{4} M^{-\frac{p-2}{2}} K_{2}^{p} \frac{\alpha^{2}}{\varrho^{2}} \\
& \quad=4 K_{1}^{2}+4 K_{2}^{2}\left(1+6 C_{4}\right) \alpha^{\frac{4}{p}} \varrho^{-\frac{4}{p}} \leq 4 K^{2}\left(1+6 C_{4}\right) \alpha^{\frac{4}{p}} \varrho^{-\frac{4}{p}} .
\end{aligned}
$$

Taking

$$
\alpha \geq C_{5}\left(1+K^{\frac{2 p}{3 p-4}}\right) \varrho^{\frac{4 p-4}{3 p-4}} \geq C_{5}\left(1+K^{\frac{2 p}{3 p-4}}\right) \varrho^{\frac{4}{3}},
$$

we can guarantee that $\alpha>C_{2}$,

$$
\frac{\alpha^{3}}{3 C_{3} \varrho^{4}} \geq 3\left(4 K^{2}\left(1+6 C_{4}\right) \alpha^{\frac{4}{p}} \varrho^{-\frac{4}{p}}\right),
$$


and

$$
\frac{\alpha}{3 C_{3} \varrho^{2}}-16 C_{4} M^{-\frac{p-2}{2}} K_{2}^{p} \geq 0
$$

Using (2.1) and recalling (1.6), we obtain

$$
\int_{\mathbb{R}^{2}} \omega_{\varrho}^{-1-2 \alpha} \eta^{2} \psi^{2} d x \geq\left(\frac{\varrho}{Q}\right)^{1+2 \alpha}\left\|\psi_{\Theta}\right\|_{2}^{2} \geq\left(2 C_{1}\right)^{1+2 \alpha}\left\|\psi_{\Theta}\right\|_{2}^{2}
$$

Combining (2.38), (2.41), (2.42) and (2.43), we conclude that

$$
\begin{aligned}
\frac{2 \alpha^{3}}{9 C_{3} \varrho^{4}}\left(2 C_{1}\right)^{1+2 \alpha}\left\|\psi_{\Theta}\right\|_{2}^{2} \leq 4 \int_{\operatorname{supp} \nabla \eta} \omega_{\varrho}^{2-2 \alpha}|\nabla \eta|^{2}|\nabla \psi|^{2} d x \\
+\int_{\operatorname{supp} \nabla \eta} \omega_{\varrho}^{2-2 \alpha}(\Delta \eta)^{2} \psi^{2} d x+2 \int_{\operatorname{supp} \eta} \omega_{\varrho}^{2-2 \alpha} \eta^{2} \zeta^{2} d x .
\end{aligned}
$$

Given $M>0$, we have

$$
\int_{\mathbb{R}^{2}} \varphi^{*}\left(\frac{\left|W_{M}\right|}{M^{-\frac{p-1}{2}} K_{2}^{p}}\right) d x \leq \int_{\mathbb{R}^{2}} \varphi^{*}\left(\frac{\left|V^{(2)}\right|^{p}}{K_{2}^{p}}\right) d x
$$

and hence, using $\left\|\left|V^{(2)}\right|^{p}\right\|_{\varphi^{*}} \leq K_{2}^{p}$, we get $\left\|W_{M}\right\|_{\varphi^{*}} \leq M^{-\frac{p-1}{2}} K_{2}^{p}$. Let $f \in \mathcal{D}(\nabla)$. Then, using (2.20), Hölder's inequality for Orlicz spaces (1.3), and the Sobolev inequality in [AT, Theorem 0.1], we get

$$
\left|\int_{\mathbb{R}^{2}} V f^{2} d x\right| \leq\left(K_{1}+M^{\frac{1}{2}}+2 C_{4} M^{-\frac{p-1}{2}} K_{2}^{p}\right)\|f\|_{2}^{2}+2 C_{4} M^{-\frac{p-1}{2}} K_{2}^{p}\|\nabla f\|_{2}^{2}
$$

Taking $M=\left(4 C_{4} K_{2}^{p}\right)^{\frac{2}{p-1}}$ (we can require $C_{4} \geq 1$ ), we get

$$
\left|\int_{\mathbb{R}^{2}} V f^{2} d x\right| \leq 4 C_{4}\left(1+K^{\frac{p}{p-1}}\right)\|f\|_{2}^{2}+\frac{1}{2}\|\nabla f\|_{2}^{2} .
$$

We have

$$
\begin{aligned}
& \int_{\left\{2 C_{1} Q \leq|x| \leq 2 C_{1} Q+1\right\}} \omega_{\varrho}^{2-2 \alpha}\left(4|\nabla \eta|^{2}|\nabla \psi|^{2}+(\Delta \eta)^{2} \psi^{2}\right) d x \\
& \quad \leq 64\left(\frac{C_{1} \varrho}{2 C_{1} Q}\right)^{2 \alpha-2} \int_{\left\{2 C_{1} Q \leq|x| \leq 2 C_{1} Q+1\right\}}\left(4|\nabla \psi|^{2}+\psi^{2}\right) d x \\
& \leq C_{6}\left(\frac{5}{4} C_{1}\right)^{2 \alpha-2} \int_{\left\{2 C_{1} Q-1 \leq|x| \leq 2 C_{1} Q+2\right\}}\left(\zeta^{2}+\left(1+K^{\frac{p}{p-1}}\right) \psi^{2}\right) d x \\
& \leq C_{6}\left(\frac{5}{4} C_{1}\right)^{2 \alpha-2}\left(\left\|\zeta_{\Omega}\right\|_{2}^{2}+\left(1+K^{\frac{p}{p-1}}\right)\left\|\psi_{\Omega}\right\|_{2}^{2}\right),
\end{aligned}
$$

where we used (2.47) and an interior estimate. Similarly,

$$
\begin{aligned}
& \int_{\left\{\frac{\delta}{4} \leq|x| \leq \frac{3 \delta}{4}\right\}} \omega_{\varrho}^{2-2 \alpha}\left(4|\nabla \eta|^{2}|\nabla \psi|^{2}+(\Delta \eta)^{2} \psi^{2}\right) d x \\
& \leq 1024 \delta^{-4}\left(4 \delta^{-1} C_{1} \varrho\right)^{2 \alpha-2} \int_{\left\{\frac{\delta}{4} \leq|x| \leq \frac{3 \delta}{4}\right\}}\left(4|\nabla \psi|^{2}+\psi^{2}\right) d x \\
& \leq C_{7} \delta^{-4}\left(4 \delta^{-1} C_{1} \varrho\right)^{2 \alpha-2} \int_{\{|x| \leq \delta\}}\left(\zeta^{2}+\left(K^{\frac{p}{p-1}}+\delta^{-2}\right) \psi^{2}\right) d x \\
& \leq C_{7} \delta^{-4}\left(16 \delta^{-1} C_{1}^{2} Q\right)^{2 \alpha-2}\left(\left\|\zeta_{\Omega}\right\|_{2}^{2}+\left(K^{\frac{p}{p-1}}+\delta^{-2}\right)\left\|\psi_{0, \delta}\right\|_{2}^{2}\right) .
\end{aligned}
$$


In addition,

$$
\int_{\operatorname{supp} \eta} \omega_{\varrho}^{2-2 \alpha} \eta^{2} \zeta^{2} d x \leq\left(4 \delta^{-1} C_{1} \varrho\right)^{2 \alpha-2}\left\|\zeta_{\Omega}\right\|_{2}^{2} \leq\left(16 \delta^{-1} C_{1}^{2} Q\right)^{2 \alpha-2}\left\|\zeta_{\Omega}\right\|_{2}^{2} .
$$

If we have

$$
\frac{\alpha^{3}}{\varrho^{4}}\left(\frac{8}{5}\right)^{2 \alpha}\left\|\psi_{\Theta}\right\|_{2}^{2} \geq C_{8}\left(1+K^{\frac{p}{p-1}}\right)\left\|\psi_{\Omega}\right\|_{2}^{2}
$$

we obtain

$$
C_{6}\left(\frac{5}{4} C_{1}\right)^{2 \alpha-2}\left(1+K^{\frac{p}{p-1}}\right)\left\|\psi_{\Omega}\right\|_{2}^{2} \leq \frac{1}{2} \frac{2 \alpha^{3}}{9 C_{3} \varrho^{4}}\left(2 C_{1}\right)^{1+2 \alpha}\left\|\psi_{\Theta}\right\|_{2}^{2},
$$

so we conclude that

$$
\begin{aligned}
& \frac{\alpha^{3}}{9 C_{3} \varrho^{4}}\left(2 C_{1}\right)^{1+2 \alpha}\left\|\psi_{\Theta}\right\|_{2}^{2} \\
& \quad \leq C_{9} \delta^{-4}\left(16 \delta^{-1} C_{1}^{2} Q\right)^{2 \alpha-2}\left(\left(K^{\frac{p}{p-1}}+\delta^{-2}\right)\left\|\psi_{0, \delta}\right\|_{2}^{2}+\left\|\zeta_{\Omega}\right\|_{2}^{2}\right) .
\end{aligned}
$$

Thus,

$$
\frac{\alpha^{3}}{\varrho^{4}} Q^{4}\left(\left(8 C_{1} Q\right)^{-1} \delta\right)^{2 \alpha+2}\left\|\psi_{\Theta}\right\|_{2}^{2} \leq C_{10}\left(\left(K^{\frac{p}{p-1}}+\delta^{-2}\right)\left\|\psi_{0, \delta}\right\|_{2}^{2}+\left\|\zeta_{\Omega}\right\|_{2}^{2}\right) .
$$

Since $\left(\frac{\delta}{Q}\right)^{5} \leq\left(\frac{1}{2}\right)^{5} \leq \frac{1}{8 C_{1}}$ by (1.8), we have

$$
\frac{\alpha^{3}}{\varrho^{4}} Q^{6}\left(\frac{\delta}{Q}\right)^{12 \alpha+14}\left\|\psi_{\Theta}\right\|_{2}^{2} \leq C_{11}\left(\left(1+K^{\frac{p}{p-1}}\right)\left\|\psi_{0, \delta}\right\|_{2}^{2}+\delta^{2}\left\|\zeta_{\Omega}\right\|_{2}^{2}\right) .
$$

To satisfy (2.40) and (2.51), we choose

$$
\alpha=C_{12}\left(1+K^{\frac{2 p}{3 p-4}}\right)\left(Q^{\frac{4 p-4}{3 p-4}}+\log \frac{\left\|\psi_{\Omega}\right\|_{2}}{\left\|\psi_{\Theta}\right\|_{2}}\right),
$$

Combining with (2.55), and recalling $Q \geq 1$, we get

$$
\begin{gathered}
\left(1+K^{\frac{2 p}{3 p-4}}\right)^{3}\left(\frac{\delta}{Q}\right)^{C_{13}\left(1+K^{\frac{2 p}{3 p-4}}\right)\left(Q^{\frac{4 p-4}{3 p-4}}+\log \frac{\left\|\psi_{\Omega}\right\|_{2}}{\left\|\psi_{\Theta}\right\|_{2}}\right)}\left\|\psi_{\Theta}\right\|_{2}^{2} \\
\leq C_{14}\left(\left(1+K^{\frac{p}{p-1}}\right)\left\|\psi_{0, \delta}\right\|_{2}^{2}+\delta^{2}\left\|\zeta_{\Omega}\right\|_{2}^{2}\right),
\end{gathered}
$$

and hence there exists $m>0$ such that

$$
\left(\frac{\delta}{Q}\right)^{m\left(1+K^{\frac{2 p}{3 p-4}}\right)\left(Q^{\frac{4 p-4}{3 p-4}}+\log \frac{\left\|\psi_{\Omega}\right\|_{2}}{\left\|\psi_{\Theta}\right\|_{2}}\right)}\left\|\psi_{\Theta}\right\|_{2}^{2} \leq\left\|\psi_{x_{0}, \delta}\right\|_{2}^{2}+\delta^{2}\left\|\zeta_{\Omega}\right\|_{2}^{2} .
$$

If $\left\|V^{(2)}\right\|_{p} \leq K_{2}<\infty$ for some $p>2$, we have $\left(\left\|\left|V^{(2)}\right|^{p^{\prime}}\right\|_{\varphi^{*}}\right)^{\frac{1}{p^{\prime}}} \leq K_{2}$ for any $p^{\prime} \in[2, p)$ since

$$
\int_{\mathbb{R}^{2}} \varphi^{*}\left(\frac{\left|V^{(2)}\right|^{p^{\prime}}}{K_{2}^{p^{\prime}}}\right) d x \leq \int_{\mathbb{R}^{2}}\left(\frac{\left|V^{(2)}\right|^{p^{\prime}}}{K_{2}^{p^{\prime}}}\right)^{\frac{p}{p^{\prime}}} d x \leq \int_{\mathbb{R}^{2}} \frac{\left|V^{(2)}\right|^{p}}{K_{2}^{p}} d x \leq 1 .
$$

We conclude that (2.58) holds with $p^{\prime}$ substituted for $p$. Letting $p^{\prime} \uparrow p$ we obtain (2.58) since $K_{2}$ is independent of $p^{\prime}$. 
(c) $d=1$ : Let $\left\|V^{(2)}\right\|_{p} \leq K_{2}$ with $p \geq 2$. Using Hölder's inequality and (2.8) with $q=2$, we get

$$
\int_{\mathbb{R}} W_{M}^{2} \omega_{\varrho}^{2-2 \alpha} \eta^{2} \psi^{2} d x \leq\left\|W_{M}\right\|_{2}^{2}\left\|\omega_{\varrho}^{2-2 \alpha} \eta^{2} \psi^{2}\right\|_{\infty} \leq M^{-\frac{p-2}{2}} K_{2}^{p}\left\|\omega_{\varrho}^{2-2 \alpha} \eta^{2} \psi^{2}\right\|_{\infty} .
$$

Applying Sobolev's inequality, we obtain

$$
\begin{aligned}
& \left\|\omega_{\varrho}^{2-2 \alpha} \eta^{2} \psi^{2}\right\|_{\infty} \leq \int_{\mathbb{R}}\left|\omega_{\varrho}^{1-\alpha} \eta \psi\right|^{2} d x+\int_{\mathbb{R}}\left|\left(\omega_{\varrho}^{1-\alpha} \eta \psi\right)^{\prime}\right|^{2} d x \\
& \leq \int_{\mathbb{R}}\left|\omega_{\varrho}^{1-\alpha} \eta \psi\right|^{2} d x+2 \int_{\mathbb{R}}\left|\left(\omega_{\varrho}^{1-\alpha}\right)^{\prime}\right|^{2} \eta^{2} \psi^{2} d x+2 \int_{\mathbb{R}} \omega_{\varrho}^{1-2 \alpha}\left|(\eta \psi)^{\prime}\right|^{2} d x .
\end{aligned}
$$

Combining (2.7), (2.60), (2.61), and (2.12) with $d=1$, we conclude that

$$
\begin{gathered}
\left(\frac{\alpha^{3}}{3 C_{3} \varrho^{4}}-4 K_{1}^{2}-4 M-4 M^{-\frac{p-2}{2}} K_{2}^{p}-8 C_{4} M^{-\frac{p-2}{2}} K_{2}^{p} \frac{\alpha^{2}}{\varrho^{2}}\right) \int_{\mathbb{R}} \omega_{\varrho}^{-1-2 \alpha} \eta^{2} \psi^{2} d x \\
+\left(\frac{\alpha}{3 C_{3} \varrho^{2}}-8 M^{-\frac{p-2}{2}} K_{2}^{p}\right) \int_{\mathbb{R}} \omega_{\varrho}^{1-2 \alpha}\left|(\eta \psi)^{\prime}\right|^{2} d x \\
\leq 4 \int_{\operatorname{supp} \eta^{\prime}} \omega_{\varrho}^{2-2 \alpha}\left|\eta^{\prime}\right|^{2}\left|\psi^{\prime}\right|^{2} d x+\int_{\operatorname{supp} \eta^{\prime}} \omega_{\varrho}^{2-2 \alpha}\left(\eta^{\prime \prime}\right)^{2} \psi^{2} d x \\
+2 \int_{\operatorname{supp} \eta} \omega_{\varrho}^{2-2 \alpha} \eta^{2} \zeta^{2} d x .
\end{gathered}
$$

Assuming $\alpha \geq \varrho$, and setting $M=K_{2}^{2} \alpha^{\frac{4}{p}} \varrho^{-\frac{4}{p}}$, we have

$$
\begin{aligned}
& 4 K_{1}^{2}+4 M+4 M^{-\frac{p-2}{2}} K_{2}^{p}+8 M^{-\frac{p-2}{2}} K_{2}^{p} \frac{\alpha^{2}}{\varrho^{2}} \\
& \leq 4 K_{1}^{2}+4 M+12 M^{-\frac{p-2}{2}} K_{2}^{p} \frac{\alpha^{2}}{\varrho^{2}}=4 K_{1}^{2}+16 K_{2}^{2} \alpha^{\frac{4}{p}} \varrho^{-\frac{4}{p}} \leq 16 K^{2} \alpha^{\frac{4}{p}} \varrho^{-\frac{4}{p}} .
\end{aligned}
$$

Taking

$$
\alpha \geq C_{5}\left(1+K^{\frac{2 p}{3 p-4}}\right) \varrho^{\frac{4 p-4}{3 p-4}} \geq C_{5}\left(1+K^{\frac{2 p}{3 p-4}}\right) \varrho^{\frac{4}{3}},
$$

we can guarantee that $\alpha>C_{2}$,

$$
\frac{\alpha^{3}}{3 C_{3} \varrho^{4}} \geq 3\left(16 K^{2} \alpha^{\frac{4}{p}} \varrho^{-\frac{4}{p}}\right)
$$

and

$$
\frac{\alpha}{3 C_{3} \varrho^{2}}-8 M^{-\frac{p-2}{2}} K_{2}^{p} \geq 0 .
$$

Using (2.1) and recalling (1.6), we obtain

$$
\int_{\mathbb{R}} \omega_{\varrho}^{-1-2 \alpha} \eta^{2} \psi^{2} d x \geq\left(\frac{\varrho}{Q}\right)^{1+2 \alpha}\left\|\psi_{\Theta}\right\|_{2}^{2} \geq\left(2 C_{1}\right)^{1+2 \alpha}\left\|\psi_{\Theta}\right\|_{2}^{2} .
$$


Combining (2.62), (2.65), (2.66) and (2.67), we conclude that

$$
\begin{aligned}
& \frac{2 \alpha^{3}}{9 C_{3} \varrho^{4}}\left(2 C_{1}\right)^{1+2 \alpha}\left\|\psi_{\Theta}\right\|_{2}^{2} \leq 4 \int_{\operatorname{supp} \eta^{\prime}} \omega_{\varrho}^{2-2 \alpha}\left|\eta^{\prime}\right|^{2}\left|\psi^{\prime}\right|^{2} d x \\
& +\int_{\operatorname{supp} \eta^{\prime}} \omega_{\varrho}^{2-2 \alpha}\left(\eta^{\prime \prime}\right)^{2} \psi^{2} d x+2 \int_{\operatorname{supp} \eta} \omega_{\varrho}^{2-2 \alpha} \eta^{2} \zeta^{2} d x
\end{aligned}
$$

Let $f \in \mathcal{D}(\nabla)$ and $M>0$. Using (2.20), Hölder's inequality, (2.8) with $d=1$, and Sobolev's inequality, we get

$$
\left|\int_{\mathbb{R}} V f^{2} d x\right| \leq\left(K_{1}+M^{\frac{1}{2}}+M^{-\frac{p-1}{2}} K_{2}^{p}\right)\|f\|_{2}^{2}+M^{-\frac{p-1}{2}} K_{2}^{p}\left\|f^{\prime}\right\|_{2}^{2} .
$$

Taking $M=\left(2 K_{2}^{p}\right)^{\frac{2}{p-1}}$, we get

$$
\left|\int_{\mathbb{R}} V f^{2} d x\right| \leq 2\left(1+K^{\frac{p}{p-1}}\right)\|f\|_{2}^{2}+\frac{1}{2}\left\|f^{\prime}\right\|_{2}^{2} .
$$

We have

$$
\begin{aligned}
& \int_{\left\{2 C_{1} Q \leq|x| \leq 2 C_{1} Q+1\right\}} \omega_{\varrho}^{2-2 \alpha}\left(4\left|\eta^{\prime}\right|^{2}\left|\psi^{\prime}\right|^{2}+\left(\eta^{\prime \prime}\right)^{2} \psi^{2}\right) d x \\
& \quad \leq 64\left(\frac{C_{1} \varrho}{2 C_{1} Q}\right)^{2 \alpha-2} \int_{\left\{2 C_{1} Q \leq|x| \leq 2 C_{1} Q+1\right\}}\left(4\left|\psi^{\prime}\right|^{2}+\psi^{2}\right) d x \\
& \quad \leq C_{6}\left(\frac{5}{4} C_{1}\right)^{2 \alpha-2} \int_{\left\{2 C_{1} Q-1 \leq|x| \leq 2 C_{1} Q+2\right\}}\left(\zeta^{2}+\left(1+K^{\frac{p}{p-1}}\right) \psi^{2}\right) d x \\
& \quad \leq C_{6}\left(\frac{5}{4} C_{1}\right)^{2 \alpha-2}\left(\left\|\zeta_{\Omega}\right\|_{2}^{2}+\left(1+K^{\frac{p}{p-1}}\right)\left\|\psi_{\Omega}\right\|_{2}^{2}\right),
\end{aligned}
$$

where we used (2.47) and an interior estimate. Similarly,

$$
\begin{aligned}
& \int_{\left\{\frac{\delta}{4} \leq|x| \leq \frac{3 \delta}{4}\right\}} \omega_{\varrho}^{2-2 \alpha}\left(4\left|\eta^{\prime}\right|^{2}\left|\psi^{\prime}\right|^{2}+\left(\eta^{\prime \prime}\right)^{2} \psi^{2}\right) d x \\
& \quad \leq 1024 \delta^{-4}\left(4 \delta^{-1} C_{1} \varrho\right)^{2 \alpha-2} \int_{\left\{\frac{\delta}{4} \leq|x| \leq \frac{3 \delta}{4}\right\}}\left(4\left|\psi^{\prime}\right|^{2}+\psi^{2}\right) d x \\
& \quad \leq C_{7} \delta^{-4}\left(4 \delta^{-1} C_{1} \varrho\right)^{2 \alpha-2} \int_{\{|x| \leq \delta\}}\left(\zeta^{2}+\left(K^{\frac{p}{p-1}}+\delta^{-2}\right) \psi^{2}\right) d x \\
& \quad \leq C_{7} \delta^{-4}\left(16 \delta^{-1} C_{1}^{2} Q\right)^{2 \alpha-2}\left(\left\|\zeta_{\Omega}\right\|_{2}^{2}+\left(K^{\frac{p}{p-1}}+\delta^{-2}\right)\left\|\psi_{0, \delta}\right\|_{2}^{2}\right) .
\end{aligned}
$$

In addition,

$$
\int_{\operatorname{supp} \eta} \omega_{\varrho}^{2-2 \alpha} \eta^{2} \zeta^{2} d x \leq\left(4 \delta^{-1} C_{1} \varrho\right)^{2 \alpha-2}\left\|\zeta_{\Omega}\right\|_{2}^{2} \leq\left(16 \delta^{-1} C_{1}^{2} Q\right)^{2 \alpha-2}\left\|\zeta_{\Omega}\right\|_{2}^{2} .
$$

If we have

$$
\frac{\alpha^{3}}{\varrho^{4}}\left(\frac{8}{5}\right)^{2 \alpha}\left\|\psi_{\Theta}\right\|_{2}^{2} \geq C_{8}\left(1+K^{\frac{p}{p-1}}\right)\left\|\psi_{\Omega}\right\|_{2}^{2}
$$

we obtain

$$
C_{6}\left(\frac{5}{4} C_{1}\right)^{2 \alpha-2}\left(1+K^{\frac{p}{p-1}}\right)\left\|\psi_{\Omega}\right\|_{2}^{2} \leq \frac{1}{2} \frac{2 \alpha^{3}}{9 C_{3} \varrho^{4}}\left(2 C_{1}\right)^{1+2 \alpha}\left\|\psi_{\Theta}\right\|_{2}^{2},
$$


so we conclude that

$$
\begin{aligned}
& \frac{\alpha^{3}}{9 C_{3} \varrho^{4}}\left(2 C_{1}\right)^{1+2 \alpha}\left\|\psi_{\Theta}\right\|_{2}^{2} \\
& \quad \leq C_{9} \delta^{-4}\left(16 \delta^{-1} C_{1}^{2} Q\right)^{2 \alpha-2}\left(\left(K^{\frac{p}{p-1}}+\delta^{-2}\right)\left\|\psi_{0, \delta}\right\|_{2}^{2}+\left\|\zeta_{\Omega}\right\|_{2}^{2}\right) .
\end{aligned}
$$

Thus,

$$
\frac{\alpha^{3}}{\varrho^{4}} Q^{4}\left(\left(8 C_{1} Q\right)^{-1} \delta\right)^{2 \alpha+2}\left\|\psi_{\Theta}\right\|_{2}^{2} \leq C_{10}\left(\left(K^{\frac{p}{p-1}}+\delta^{-2}\right)\left\|\psi_{0, \delta}\right\|_{2}^{2}+\left\|\zeta_{\Omega}\right\|_{2}^{2}\right) .
$$

Since $\left(\frac{\delta}{Q}\right)^{5} \leq\left(\frac{1}{2}\right)^{5} \leq \frac{1}{8 C_{1}}$ by (1.8), we have

$$
\frac{\alpha^{3}}{\varrho^{4}} Q^{6}\left(\frac{\delta}{Q}\right)^{12 \alpha+14}\left\|\psi_{\Theta}\right\|_{2}^{2} \leq C_{11}\left(\left(1+K^{\frac{p}{p-1}}\right)\left\|\psi_{0, \delta}\right\|_{2}^{2}+\delta^{2}\left\|\zeta_{\Omega}\right\|_{2}^{2}\right) .
$$

To satisfy (2.64) and (2.74), we choose

$$
\alpha=C_{12}\left(1+K^{\frac{2 p}{3 p-4}}\right)\left(Q^{\frac{4 p-4}{3 p-4}}+\log \frac{\left\|\psi_{\Omega}\right\|_{2}}{\left\|\psi_{\Theta}\right\|_{2}}\right)
$$

Combining with (2.78), and recalling $Q \geq 1$, we get

$$
\begin{gathered}
\left(1+K^{\frac{2 p}{3 p-4}}\right)^{3}\left(\frac{\delta}{Q}\right)^{C_{13}\left(1+K^{\frac{2 p}{3 p-4}}\right)\left(Q^{\frac{4 p-4}{3 p-4}}+\log \frac{\left\|\psi_{\Omega}\right\|_{2}}{\left\|\psi_{\Theta}\right\|_{2}}\right)}\left\|\psi_{\Theta}\right\|_{2}^{2} \\
\leq C_{14}\left(\left(1+K^{\frac{p}{p-1}}\right)\left\|\psi_{0, \delta}\right\|_{2}^{2}+\delta^{2}\left\|\zeta_{\Omega}\right\|_{2}^{2}\right),
\end{gathered}
$$

and hence there exists $m>0$ such that

$$
\left(\frac{\delta}{Q}\right)^{m\left(1+K^{\frac{2 p}{3 p-4}}\right)\left(Q^{\frac{4 p-4}{3 p-4}}+\log \frac{\left\|\psi_{\Omega}\right\|_{2}}{\left\|\psi_{\Theta}\right\|_{2}}\right)}\left\|\psi_{\Theta}\right\|_{2}^{2} \leq\left\|\psi_{x_{0}, \delta}\right\|_{2}^{2}+\delta^{2}\left\|\zeta_{\Omega}\right\|_{2}^{2}
$$

\section{UNIQUE CONTINUATION PRINCIPLE FOR SPECTRAL PROJECTIONS OF SCHRÖDINGER OPERATORS WITH SINGULAR POTENTIALS}

The following theorem, a consequence of Theorem 1.1, is an extension of [KlN, Theorem B.4] to Schrödinger operators with singular potentials. Theorem 1.2 follows from Theorem 3.1 .

Theorem 3.1. Let $H=-\Delta+V$ be a Schrödinger operator on $\mathrm{L}^{2}\left(\mathbb{R}^{d}\right)$, where $V=V^{(1)}+V^{(2)}$ with $\left\|V^{(1)}\right\|_{\infty} \leq K_{1}<\infty$ and $\left\|V^{(2)}\right\|_{p} \leq K_{2}<\infty$ with $p \geq d$ for $d \geq 3, p>2$ for $d=2$, and $p \geq 2$ for $d=1$. Set $K=K_{1}+K_{2}$. Fix $\delta \in\left(0, \frac{1}{2}\right]$, let $\left\{y_{k}\right\}_{k \in \mathbb{Z}^{d}}$ be sites in $\mathbb{R}^{d}$ with $B\left(y_{k}, \delta\right) \subset \Lambda_{1}(k)$ for all $k \in \mathbb{Z}^{d}$. There exists a constant $M_{d}>0$, such that given a rectangle $\Lambda$ as in (1.11), where $a \in \mathbb{R}^{d}$ and $L_{j} \geq 114 \sqrt{d}$ for $j=1, \ldots, d$, and a real-valued $\psi \in \mathcal{D}\left(H_{\Lambda}\right)$, we have

$$
\left.\delta^{M_{d}\left(1+K^{\beta} d, p\right.}\right)\left\|\psi_{\Lambda}\right\|_{2}^{2} \leq \sum_{k \in \mathbb{Z}^{d}, \Lambda_{1}(k) \subset \Lambda}\left\|\psi_{y_{k}, \delta}\right\|_{2}^{2}+\delta^{2}\left\|((-\Delta+V) \psi)_{\Lambda}\right\|_{2}^{2},
$$

where

$$
\beta_{d, p}=\left\{\begin{array}{lll}
\frac{2 p}{3 p-2 d} & \text { for } & d \geq 2 \\
\frac{2 p}{3 p-4} & \text { for } & d=1
\end{array} .\right.
$$


Proof of Theorem 3.1. Under the hypotheses of the theorem $V \in \mathrm{L}_{\text {loc }}^{2}\left(\mathbb{R}^{d}\right)$, which implies that $\mathcal{D}\left(\Delta_{\Lambda}\right) \cap\left\{\phi \in \mathrm{L}^{2}(\Lambda): V \phi \in \mathrm{L}^{2}(\Lambda)\right\}$ is an operator core for $H_{\Lambda}$, so it suffices to prove the theorem for $\psi \in \mathcal{D}\left(\Delta_{\Lambda}\right)$ with $V \psi \in \mathrm{L}^{2}(\Lambda)$.

Using the notation in the proof of [KIN, Theorem B.4], we have $\left\|\widehat{V^{(1)}}\right\|_{\infty}=$ $\left\|V^{(1)}\right\|_{\infty} \leq K_{1}$ and $\left\|\widehat{V^{(2)}} \Lambda_{Y \tau}(\kappa)\right\|_{p} \leq 3^{d}\left\|V_{\Lambda}^{(2)}\right\|_{p} \leq 3^{d} K_{2}$ for any $\kappa \in \Lambda$, since $\Lambda_{Y \tau}(\kappa) \subset \Lambda_{3 \mathbf{L}}$ as $Y \tau_{j}<\frac{L_{j}}{2}, j=1,2, \ldots, d$. Using Theorem 1.1 and following the proof of [KIN, Theorem B.4], we prove (3.1).

Proof of Theorem 1.2. From (2.22), (2.47) and (2.70), there exists a constant $C_{d}>$ 0 such that for all $f \in \mathcal{D}(\nabla)$

$$
\left|\int_{\mathbb{R}^{d}} V f^{2} d x\right| \leq \theta\|f\|_{2}^{2}+\frac{1}{2}\|\nabla f\|_{2}^{2}
$$

where $\theta=C_{d}\left(1+K^{\frac{2 p}{2 p-d}}\right)$ for $d \geq 2$ and $\theta=C_{1}\left(1+K^{\frac{p}{p-1}}\right)$ for $d=1$. Therefore $\sigma\left(H_{\Lambda}\right) \subset[-\theta, \infty)$, and hence it suffices to consider $E_{0} \geq-\theta$ and $E \in\left[-\theta, E_{0}\right]$. We have $V-E=\left(V^{(1)}-E\right)+V^{(2)}$, where

$$
\left\|V^{(1)}-E\right\|_{\infty} \leq\left\|V^{(1)}\right\|_{\infty}+\max \left\{E_{0}, \theta\right\} \leq K_{1}+E_{0}+\theta
$$

and $\left\|V^{(2)}\right\|_{p} \leq K_{2}$. Applying Theorem 3.1 and following the proof of KIN, Theorem B.1], we prove (1.13).

\section{REFERENCES}

[AT] Adachi, S., Tanaka, K.: Trudinger type inequalities in $R^{N}$ and their best exponents Proc. Amer. Math. Soc., 128, no. 7, 2051-2057 (1999)

[BK] Bourgain, J., Kenig, C.: On localization in the continuous Anderson-Bernoulli model in higher dimension, Invent. Math. 161, 389-426 (2005)

[BKl] Bourgain, J., Klein, A.: Bounds on the density of states for Schrödinger operators. Invent. Math. 194, 41-72 (2013)

[CHK1] Combes, J.M., Hislop, P.D., Klopp, F.: Hölder continuity of the integrated density of states for some random operators at all energies. IMRN 4, 179-209 (2003)

[CHK2] Combes, J.M., Hislop, P.D., Klopp, F.: Optimal Wegner estimate and its application to the global continuity of the integrated density of states for random Schrödinger operators. Duke Math. J. 140, 469-498 (2007)

[EV] Escauriaza, L., Vessella, S.: Optimal three cylinder inequalities for solutions to parabolic equations with Lipschitz leading coefficients. In: Inverse Problems: Theory and Applications, Cortona/Pisa, 2002. Contemp. Math., vol. 333,79-87. Amer. Math. Soc., Providence (2003)

[GK1] Germinet, F., Klein, A.: A characterization of the Anderson metal-insulator transport transition. Duke Math. J. 124, 309-351 (2004)

[GK2] Germinet, F., Klein, A.: A comprehensive proof of localization for continuous Anderson models with singular random potentials. J. Eur. Math. Soc. 15, 53-143 (2013)

[GiT] Gilbarg, D., Trudinger, N.: Elliptic Partial Differential Equations of Second Order. Classics in Mathematics. Springer, Berlin (2001). Reprint of the 1998 edition

[KSU] Kenig, C. E., Salo, M., Uhlmann, G.: Inverse problems for the anisotropic Maxwell equations, Duke Math. J. 157 369-419 (2011)

[Kl] Klein, A.: Unique continuation principle for spectral projections of Schrödinger operators and optimalWegner estimates for non-ergodic random Schrödinger operators. Comm. Math Phys. 323, 1229-1246 (2013)

[KlN] Klein, A., Nguyen, S.: Bootstrap multiscale analysis and localization for the multi-particle continuous Anderson Hamiltonian, J. Spectr. Theory, to appear

[RR] Rao, M. M., Ren, Z. D.: Theory of Orlicz spaces. M. Dekker, Inc., New York (1991)

$[\mathrm{RoV}]$ Rojas-Molina, C., Veselić, I.: Scale-free unique continuation estimates and applications to random Schrödinger operators. Commun. Math. Phys. 320, 245-274 (2013) 
University of California, Irvine, Department of Mathematics, Irvine, CA 92697-3875, USA

E-mail address, Klein: aklein@uci.edu

E-mail address, Tsang: tsangcs@uci.edu 